\title{
Interleukin-6: A Proinflammatory Role in Nephritis in Patients with Systemic Lupus Erythematosus
}

\author{
Dalia Abdul-Halim Shaheen ${ }^{1}$, Hisham M. Habib ${ }^{2}$, Mohamed A. Marie ${ }^{3}$ \\ ${ }^{1}$ Department of Medical Biochemistry, Faculty of Medicine, Mansoura University, Cairo, Egypt \\ ${ }^{2}$ Department of Rheumatology and Rehabilitation, Faculty of Medicine, Mansoura University, Cairo, Egypt \\ ${ }^{3}$ Department of Internal Medicine, Faculty of Medicine, Cairo University, Cairo, Egypt
}

Email address:

daliashaheen72@yahoo.com (D. Abdul-Halim Shaheen)

\section{To cite this article:}

Dalia Abdul-Halim Shaheen, Hisham M. Habib, Mohamed A. Marie. Interleukin-6: A Proinflammatory Role in Nephritis in Patients with Systemic Lupus Erythematosus. International Journal of Genetics and Genomics. Vol. 3, No. 5, 2015, pp. 53-58.

doi: $10.11648 /$ j.ijgg.20150305.13

\begin{abstract}
Background. Systemic lupus erythematosus (SLE) is a systemic autoimmune disease with a common finding of renal involvement which is related to high incidence of mortality and morbidity. IL-6 is produced by leukocytes and intrinsic kidney cells that affects inflammation, increases mesangial cell proliferation, and also contributes to autoimmunity. Objective. To detect the serum level as well as expression in PBMCs in the blood of IL-6 in SLE patients. Furthermore we compare serum level, as well as expression in PBMCs in the blood of IL-6 of lupus nephritis group versus non-nephritis lupus group and to study its correlation with other variables in SLE patients. Methods. The study was carried on 100 SLE patients, and 50 healthy control subjects. Fifty patients had lupus nephritis and 50 without evidence of lupus nephritis. Serum interleukin 6 (IL6) was measured using the ELISA technique as well as the expression of IL6 mRNA in PBMCs. Serum creatinine, C3, C4 and 24 hours urinary proteins were measured. Lupus activity was assessed using BILAG scoring system. Renal activity was measured using renal activity scoring system. Results. Serum IL6 level and its mRNA expression was significantly high in SLE patients and was higher in lupus nephritis patients than lupus patients without nephritis. IL6 was significantly correlated with renal activity score, 24 hours urinary proteins lowered C3 and C4 level and BILAG score. Conclusion. Serum IL-6 level and its mRNA expression is elevated in SLE patients as well as lupus nephritis patients. This was found to be linked with SLE disease activity in general and renal involvement in particular.
\end{abstract}

Keywords: Autoimmune, Cytokines, IL 6, SLE

\section{Introduction}

Systemic lupus erythematosus (SLE) is a systemic autoimmune disease with a common finding of renal involvement that is related to high incidence of mortality and morbidity [1]. Histologically, renal lupus involvement shows a broad range of patterns, which cause assorted clinical manifestations, ranging from mild asymptomatic urinary test abnormalities to severe nephrotic syndrome or rapidly progressive renal failure [2]. Immunopathogenesis involves formation of Immune complexes, provoking aberrant production of type I interferons $\alpha$ and $\beta$ (IFN- $\alpha / \beta)$ and proinflammatory cytokines $[3,4]$. Lupus nephritis results from a complex interaction between autoantibodies in association with anti-dsDNA, nucleosomes and histones that end up forming kidney immune complexes and permanently activated inflammatory cells that stimulate and induce proliferation in local cells, which, in turn, stimulate complement, cytokines and chemokines [5].

Chemokines can amplify kidney inflammation by recruiting more leukocytes to the kidney in a positivefeedback loop. Cytokines can damage kidney parenchyma directly. For example, in murine lupus nephritis, exogenous IL-6 enhanced the progression of kidney injury, whereas treatment with an anti-IL-6 monoclonal antibody alleviated disease [6]. Cytokines also can mediate deleterious processes such as mesangial and glomerular epithelial cell proliferation, glomerulosclerosis, tubular atrophy, and interstitial fibrosis [7]. IL-6 is a multifunctional cytokine produced by leukocytes and intrinsic kidney cells that affects inflammation, increases mesangial cell proliferation, and also contributes to autoimmunity by stimulating terminal B-cell differentiation, autoantibody secretion, and T-cell 
differentiation [8, 9]. IL-6 works in synergy with IL-1 and tumor necrosis factor (TNF) $\alpha$ to promote inflammation, but also can be anti-inflammatory by termination of IL-1- and TNF- $\alpha-$ mediated inflammatory cascades [10].

In spite of extensive research, there are still unanswered questions regarding the role of inflammation in the pathogenesis of renal damage in systemic lupus erythematosus [11-13] and furthermore the implication of IL6 in the initiation of inflammatory cascade. The aim of current study was to detect the serum level of IL-6 in SLE patients. We plan to examine the expression of IL-6 in blood of SLE patients. Furthermore, we compare serum level of IL6 between nephritis and non-nephritis groups and to study its correlation with other variables including 24 hour urinary proteins, BILAG score, $\mathrm{C} 3, \mathrm{C} 4$, serum creatinine, and renal activity score.

\section{Material and Methods}

\section{Patients}

In our case control study, we recruited 100 SLE patients diagnosed as having systemic lupus erythematosus who fulfilled the American College of Rheumatology (ACR) classification criteria for SLE [14]. Patients were collected from departments of rheumatology, nephrology and internal medicine at $\mathrm{Al}$ ahsa Hospital, in eastern area of Saudi Arabia, from January 2013 to January 2015. Patients with a previous diagnosis of another connective tissue disorder which may associates SLE such as rheumatoid arthritis, primary Sjogren's syndrome, mixed connective tissue disease, systemic sclerosis, antiphospholipid antibody syndrome or idiopathic inflammatory myositis were excluded from this study. Also, patients with liver disease, other autoimmune disease, or malignancies were excluded. This aimed to avoid the possible effects of these comorbid conditions on cytokine production. Patients were divided into two groups. Group (1): involved 50 patients with evidence of lupus nephritis as having 24 hour urinary protein $>0.5$ gm or active urinary sediment in the form of either microscopic or macroscopic hematuria or white cell cast with or without elevated serum creatinine. Group (2): involved 50 SLE patients without any evidence of lupus nephritis. Group (3): Fifty healthy age- and sex-matched control subjects were volunteered to act as a healthy control group (HCs). All patients were subjected to careful history taking, full clinical examination and routine laboratory investigations.

\section{Clinical assessment}

Overall clinical assessment and disease activity index of SLE patients was done using the BILAG (British Isles Lupus Assessment Group) disease activity index [15]. It distinguishes activity in 8 organs or systems namely general, mucocutaneous, central nervous system, musculoskeletal, cardio-vascular system/respiratory, vasculitis, renal and haematological systems. It provides an accurate means of grading disease activity from the "most active" to "no evidence of disease activity currently". Patients are classified to 5 grades: $A=9$ points, $\mathrm{B}=3$ points, $\mathrm{C}=1$ point, $\mathrm{D}=0$ point, $\mathrm{E}=0$ point.
The protocol for the research project has been approved by a suitably constituted Ethics Committee of the hospital and that it conforms to the provisions of the World Medical Association's Declaration of Helsinki. An informed consent has been obtained for all investigations on subjects of the study (and patient anonymity has been preserved).

\section{Laboratory investigations:}

A complete haemogram, chemistry, urinalysis, 24 hours urinary proteins and serological tests (C3 and $\mathrm{C} 4)$ were performed for patients.

Ten milliliters of venous blood was collected from each subject and divided into three samples. The first one was used for blood chemistry analysis $\mathrm{C} 3, \mathrm{C} 4$, serum creatinine. The other blood sample was used for immunoassay of serum human IL6 levels. The third one was used for expression of Human IL 6 from PMNC.

Serum creatinine was determined by an automated method based on the alkaline picrate reaction, in a Hitachi 912 (Roche) chemistry analyzer and the results were expressed as $\mathrm{mg} / \mathrm{dl}$.

Serum C3 and C4 concentrations were measured by the turbidimetric immunoassay.

A 24-hour urine sample were collected and kept in the refrigerator or a cool place during the collection period.

\section{ELISA}

Human IL-6 Immunoassay: The plasma concentration of IL6 was measured by a commercially available enzyme-linked immunosorbent assay Quantikine HS (R\&D Systems,) [16]. The level of serum IL6 of nephritis and non-nephritis groups will be correlated with the laboratory values including 24 hour urinary proteins, BILAG score, $\mathrm{C} 3, \mathrm{C} 4$, serum creatinine, and renal activity score

Real-time PCR

Total RNA was extracted from PBMC using TRIzol (Invitrogen, Carlsbad, CA, USA). (QIAamp RNA Blood Mini Kit, QIAGEN, Hilden, Germany; Cat. No. 52304) according to the manufacturer instructions.

Thereafter, RNA was reversed transcribed from $2.5 \mu \mathrm{g}$ total RNA according to the manufacturer's instructions, using random hexamers (Invitrogen). cDNA was used for real-time PCR with the help of Step One ${ }^{\mathrm{TM}}$ real-time PCR system (Applied Biosystems, Foster City, CA, USA).

The sequences of oligonucleotide primers used in the PCR for detection of IL 6 expression levels, were as follows:

- For Human IL-6: Forward is 5'AACCTGAACCTTCCAAAGATGG-3' and Reverse is 5'- TCTGGCTTGTTCCTCACTACT-3'

- For Human GAPDH sense: Forward 5'AGAAGGCTGGGGCTCATTTG-3' and Reverse 5'AGGGGCCATCCACAGTCTTC-3'.

The amplification scheme used was as follows: initial denaturation $30 \mathrm{sec}$ at $95^{\circ} \mathrm{C}$, followed by 40 cycles of $5 \mathrm{sec}$ at $95^{\circ} \mathrm{C}$, and $30 \mathrm{sec}$ at $60^{\circ} \mathrm{C}$. GAPDH was used as a housekeeping gene for normalizing the expression data. Data from the reaction were collected and analyzed. The relative copy numbers of gene expression were quantitated using the comparative threshold cycle (ct) method $(\Delta \Delta \mathrm{CT})$

Renal activity score 
Renal activity score was measured using the following criteria: proteinuria $0.5-1 \mathrm{gm} /$ day $=3$ points, proteinuria $>1-3$ $\mathrm{gm} /$ day $=5$ points, proteinuria $>3 \mathrm{gm} /$ day $=11$ points, urine red blood cell count $>/=5 / \mathrm{hpf}=3$ points, urine white blood cell count $>/=5 / \mathrm{hpf}=1$ point $[17]$

Statistical analysis

Statistical analyses were performed with SPSS software (SPSS, Chicago IL, USA). Data were expressed as mean \pm SD. Differences between two groups were detected with Student's t test for parametric data and Mann-Whitney U test for nonparametric data. The Pearson correlation test was used to evaluate the correlation between serum IL- 6 levels and laboratory values and clinical features. $\mathrm{P}$ values $<0.05$ was considered as significant.

\section{Results}

\subsection{Study Population}

Demographic, clinical, and some relevant laboratory characteristics of the patients are summarized in tables (1).
The study group comprised 100 SLE patients with a mean age of $25 \pm 3.5$ years that ranged from 19.5 to 32.5 years. Eighty eight patients were females and 12 were males. Mean disease duration was $7 \pm 4.5$ (range 1 - 15) years. Then, SLE patients were divided into 2 groups; group with lupus nephritis and other group without evidence of lupus nephritis No statistically significant difference in age, gender and disease duration in both groups. As regards disease activity, there was a statistically significant increase in mean BILAG score in lupus nephritis group $(15+3.8)$ versus non-nephritis lupus group $(5+2.6)$ (p 0.005). In lupus nephritis group, mean renal activity score was $8.8+2.1$. A statistically significant increase in the level of 24 urinary protein $(2.1+1$ vs $0.24+0.08 \mathrm{~g})$, serum creatinine $(1.5+0.3$ vs $0.72+0.1 \mathrm{mg} \%)$ in lupus nephritis group versus non-nephritis lupus group ( $\mathrm{p}$ $0.01)$. A statistically significant decrease in the level of $\mathrm{C} 3$ (33.6+9 vs $117+9.8 \mathrm{mg} \%), \mathrm{C} 4(5.1+1.2$ vs $19.9+2.2 \mathrm{mg} \%)$ in lupus nephritis group versus non-nephritis lupus group ( $\mathrm{p}$ $0.01)$.

Table (1). Demographic, laboratory and activity score data in lupus nephritis patients versus non-nephritis lupus patients.

\begin{tabular}{lllll}
\hline Variables & Lupus nephritis $\mathbf{N}=\mathbf{5 0}$ & Non- nephritis lupus $\mathbf{N}=\mathbf{5 0}$ & $\mathbf{C o n t r o l s ~} \mathbf{N}=\mathbf{5 0}$ & $\mathbf{P}$ \\
\hline Age & $23.6+2.2$ & $24.4+3$ & $23.2+1.8$ & $0.07 \mathrm{NS}$ \\
Gender & & & \\
Male & $8(16 \%)$ & $4(8 \%)$ & $5(10 \%)$ & $0.08 \mathrm{NS}$ \\
Female & $42(84 \%)$ & $46(92 \%)$ & $45(90 \%)$ & $>0.1 \mathrm{NS}$ \\
Disease duration (years) & $6.5+2.4$ & $6.8+4.3$ & - & $0.005 \mathrm{~S}$ \\
BILAG Score & $15+3.8$ & $(5+2.6)$ & - & - \\
Renal Activity Score & $8.8+2.1$ & - & - & $0.01 \mathrm{~S}$ \\
24 urinary protein (g) & $2.1+1$ & $0.24+0.08$ & $0.11+0.03$ & $0.01 \mathrm{~S}$ \\
Creatinine (mg \%) & $1.5+0.3$ & $0.72+0.1$ & $0.59+0.1$ & $0.01 \mathrm{~S}$ \\
C3 (mg \%) & $33.6+9$ & $117+9.8$ & $108+13$ & $0.01 \mathrm{~S}$ \\
C4 (mg \%) & $5.1+1.2$ vs & $19.9+2.2$ & $20.6+2.8$ & \\
\hline
\end{tabular}

\subsection{Increased Serum Levels of IL-6 in SLE Patients}

Serum levels of circulating IL-6 were significantly higher in SLE patients $(40.66 \pm 22.8 \mathrm{pg} / \mathrm{ml})$ compared to $\mathrm{HC}$ subjects $(2.32 \pm 3.41 \mathrm{pg} / \mathrm{ml}) .(\mathrm{P}<0.005)$ (table 2$)$.

Table (2). Serum levels of IL-6 in SLE patients.

\begin{tabular}{lll}
\hline Group & Serum IL-6 $(\mathbf{p g} / \mathbf{m l})$ Mean \pm SD & P \\
\hline SLE $(\mathrm{N}=100)$ & $40.66 \pm 22.8$ & \\
$\mathrm{HC}(\mathrm{N}=50)$ & $2.32 \pm 3.41$ & $\mathrm{P}<0.005$ \\
\hline
\end{tabular}

SLE, systemic lupus erythematosus; HC, healthy control

\subsection{Increased Serum Levels of IL-6 in Lupus Nephritis Patients Versus Non-Nephritis Lupus Group}

Table (3). Serum levels of IL-6 in lupus nephritis group versus non-nephritis lupus group.

\begin{tabular}{lll}
\hline Group & Serum IL-6 $(\mathbf{p g} / \mathbf{m l})$ Mean \pm SD & P \\
\hline lupus nephritis $(\mathrm{N}=50)$ & $70.5+25$ & \\
non-nephritis lupus $(\mathrm{N}=50)$ & $20.6+12$ & $\mathrm{P}<0.005$ \\
\hline
\end{tabular}

A statistically significant elevation in the level of IL-6 in lupus nephritis group versus non-nephritis lupus group
$(70.5+25 \mathrm{vs} 20.6+12 \mathrm{pg} / \mathrm{ml})(\mathrm{p}<0.005)($ table 3$)$.

\subsection{Upregulated Expression of IL-6 in PBMCs from SLE Patients}

Expression of IL-6 mRNA was significantly higher in SLE PBMCs when compared to HC (122 \pm 15.8 vs $31.37 \pm 9.5$; respectively) (p 0.01) (table 4).

Table (4)-A. Serum IL-6 $m R N A$ relative expression in SLE patients.

\begin{tabular}{lll}
\hline Group & IL-6 mRNA Mean \pm SD & P \\
\hline SLE $(\mathrm{N}=100)$ & $122 \pm 15.8$ & \\
$\mathrm{HC}(\mathrm{N}=50)$ & $31.37 \pm 9.5$ & P 0.01 \\
\hline
\end{tabular}

SLE, systemic lupus erythematosus; HC, healthy control.

\subsection{Upregulated Expression of IL-6 in Peripheral Blood Mononuclear Cells (PBMCs) from Lupus Nephritis Group Versus Non-Nephritis Lupus Group}

Expression of IL-6 mRNA was significantly higher in PBMCs of lupus nephritis group versus non-nephritis lupus group (142 \pm 22.8 vs $91.37 \pm 9.5$; respectively) (p 0.05) (table $5)$. 
Table (5)-A. IL-6 mRNA relative expression in peripheral blood mononuclear cells (PBMCs) from lupus nephritis group versus non-nephritis lupus group.

\begin{tabular}{lll}
\hline Group & IL-6 mRNA Mean \pm SD & P \\
\hline lupus nephritis $(\mathrm{N}=50)$ & $142 \pm 22.8$ & \\
non-nephritis lupus $(\mathrm{N}=50)$ & $91.37 \pm 9.5$ & $\mathrm{P}<0.05$ \\
\hline
\end{tabular}

\subsection{Correlation Between Serum Levels of IL-6 Versus Other Variables Among Lupus Nephritis Group and Non-Nephritis Lupus Group}

Tables (6) and (7) demonstrate correlation between IL-6 versus other variables among lupus nephritis group and nonnephritis lupus group, respectively. In lupus nephritis group, there was a statistically significant positive correlation between IL-6 versus 24 urinary protein level, BILAG score, renal activity score, while there was an inverse correlation between IL- 6 versus $\mathrm{C} 3$ and $\mathrm{C} 4$. In non-nephritis lupus group, there was no any significant correlation between IL-6 versus other variables.

Table (6). Correlation between serum levels of IL-6 versus other variables among lupus nephritis group.

\begin{tabular}{lll}
\hline IL-6 & & Variables \\
\hline $\mathbf{P}$ & $\mathbf{r}$ & \\
\hline 0.01 & 0.82 & 24 urinary protein \\
0.01 & 0.55 & BILAG score \\
0.01 & -0.39 & C3 \\
0.01 & -0.45 & C4 \\
0.1 & 0.08 & Creatinine \\
0.01 & 0.59 & Renal Activity Score \\
\hline
\end{tabular}

BILAG, British Isles Lupus Assessment Group; C3, complement 3; C4, complement 4

Table (7). Correlation between serum levels of IL-6 versus other variables among non nephritis lupus group.

\begin{tabular}{lll}
\hline $\mathbf{1 L}-6$ & & Variables \\
\hline $\mathbf{P}$ & $\mathbf{r}$ & 24 urinary protein \\
0.5 & 0.11 & BILAG \\
0.07 & 0.13 & C3 \\
0.5 & -0.17 & C4 \\
0.1 & -0.12 & Creatinine \\
0.1 & 0.18 & Renal Activity Score \\
0.08 & 0.19 & \\
\hline
\end{tabular}

BILAG, British Isles Lupus Assessment Group; C3, complement 3; C4, complement 4

\section{Discussion}

Systemic Lupus Erythematosus (SLE) is an inflammatory, multisystem, autoimmune disease characterized by a wide variety of autoantibodies, some of which are pathogenic. In latest years it has become more evident that the polyclonal B cell activation in SLE is T-cell dependent. The stimulation of the autoantibody producing B cells is likely facilitated by the TH2 subtype of T cells producing IL-4, IL-5, IL-6 and IL- 10, whereas the TH1 subtype secreting IL-2 and IFN-gamma predominates in cell-mediated immune response [18]. Tumor necrosis factor (TNF)- $\alpha$ is not just a proinflammatory cytokine. It has also been proposed to be an immunoregulatory molecule that can alter the balance of $\mathrm{T}$ regulatory cells [19]. TNF- $\alpha$ has differential effects on B cells, on $\mathrm{T}$ cells and on dendritic cells as well as on the process of programmed cell death. Understanding how the immune system integrates the pleiotropic properties of TNF$\alpha$ is a contest, mostly so in diseases like SLE. Meanwhile the role of IL-6 in the pathogenesis of SLE is controversial [18].

The current study addresses the serum level of IL- 6 as well as its expression in blood of a cohort of SLE patients. We, then compare the serum level of IL-6 in lupus nephritis versus non lupus nephritis patients. Finally, we tried to disclose any correlation between the serum level of IL-6 SLE patients with other variables including 24 hour urinary proteins, BILAG score, $\mathrm{C} 3, \mathrm{C} 4$, serum creatinine, and renal activity score.

We found a significant higher serum level of IL-6 in SLE patients. Moreover, IL-6 is expressed in blood of SLE in a significant high manner since the mRNA levels of IL-6 in PBMC were significantly higher in SLE than those in HC. Furthermore, we found a statistically significant elevation in the level of IL-6 in lupus nephritis group versus nonnephritis lupus group. Finally, a significant direct correlation was found between serum level of IL-6 with 24 hour urinary protein, BILAG score for disease activity, renal activity score and inverse correlation with $\mathrm{C} 3$ and $\mathrm{C} 4$ lupus nephritis group. On the other hand, in non-nephritis lupus patients, no correlation was detected with any of these variables. This correlation appeared to be logic in respect of linking IL-6 with diseased activity in general and renal involvement in particular.

It postulated that, clinically evident renal disease occurs in approximately half of the patients with SLE [20]. Pathogenesis of lupus nephritis is multifactorial, most importantly immune complex deposition [21]. In the normal kidney, production of inflammatory chemokines is low, but is significantly increased under pathophysiological circumstances such as ischemia, toxin exposure, or acute inflammation [22].

IL-6 is a pleiotropic cytokine expressed by antigenpresenting cells. Production of IL-6 is induced by IL-1, TNF$\alpha$, lipopolysaccharide, and platelet-derived growth factor (PDGF) [23]. IL-6 induces B-cell differentiation into antibody-producing cells, T-cell differentiation into effector cells, and also induces the production of many acute phase proteins [24-26]. Richards et al. initially showed that IL-6deficient $\mathrm{BALB} / \mathrm{c}$ mice with pristine-induced lupus had lower titers of anti-dsDNA antibodies when compared to IL6 intact mice, suggesting a possible role for IL-6 in the pathogenesis of lupus nephritis and disease flares [27]. Moreover, Chemokines can amplify kidney inflammation by enrolling more leukocytes to the kidney in a positivefeedback loop. Cytokines can damage kidney parenchyma directly. For example, in murine lupus nephritis, exogenous IL-6 accelerated the progression of kidney injury, whereas treatment with an anti-IL-6 monoclonal antibody mitigated disease $[28,29]$.

Several previous studies tried to investigate levels of IL-6 
in SLE patients. Crow stated that Inflammatory cytokines, such as IL6, IL10, interferon $\alpha$ (IFN $\alpha$ ), and IL12, are elevated in the sera of SLE patients [30]. Sabry et al concluded that Serum TNF- $\alpha$ and IL- 6 are sensitive markers of SLE disease activity. They may be useful independent markers for prediction of SLE disease activity and to differentiate normal subjects from those having SLE [18]. Wei et al reported that addition of IL-6 has been shown to increase autoantibodies, such as anti-double-stranded DNA (anti-dsDNA) antibody production, making significant contributions to the inflammatory response and accelerating the progression of autoimmune diseases such as systemic lupus erythematosus [31]. Also Grondal etal. reported that serum levels of IL-6 are elevated in human SLE and have correlated with disease activity or anti-dsDNA levels in some studies [32]. The increased frequency of IL-6-producing peripheral blood mononuclear cells correlates with disease severity/activity and treatment response $[33,34]$. A blockade of IL-6 or its receptor has been demonstrated to prevent elevated anti-dsDNA antibody levels and the progression of autoimmune diseases in addition to improving survival rates [35]. Ball et al concluded that plasma IL-6 levels correlate with clinical and ultrasound measures of arthritis in patients with systemic lupus erythematosus with correlation to disease activity [36].

It has been reported that serum and urinary IL-6 levels are increased in patients with lupus nephritis, especially in those with diffuse proliferative lupus nephritis, and correlate with nephritic flares [37]. In the normal kidney, IL-6 is localized to the mesangial area and within vascular walls. In patients with lupus nephritis, its expression is increased in mesangial cells, induced in podocytes, and is present in glomerular immune deposits and along the apical aspects of proximal renal tubular epithelial cells [38-39]. Moreover, in murine and human SLE, IL-6 levels are increased in serum, urine, and glomeruli and correlate with disease activity [40]. Neutralizing IL-6 or blocking the IL-6 receptor mitigates disease in murine models of lupus nephritis [41].

Yung et al, in their research, explained the mechanisms through which IL-6 is locally produced in the kidney during pathogenesis of lupus nephritis [42]. They demonstrated that human polyclonal anti-dsDNA antibodies bind to annexin II on the surface of human mesangial cells and are rapidly internalized to induce downstream inflammatory processes including increased transcription and translation of IL-6, mediated through increased activation of ERK and p38 MAPK. Following binding and internalization, the subsequent cellular localization of anti-dsDNA antibodies can influence the amount of IL- 6 secreted by mesangial cells. In this respect, induction of IL-6 secretion is more prominent in cells stimulated with anti-dsDNA antibodies with intranuclear localization compared to antibodies that are localized solely to the cytoplasm, and this mechanism of IL-6 induction occurs with autoantibodies derived from patients in remission and with relapse. The importance of anti-dsDNA antibody-annexin II interaction in the induction of IL-6 secretion was corroborated in annexin II gene silencing studies.
Finally, we can conclude that, serum IL-6 level and its mRNA expression is elevated in SLE patients as well as lupus nephritis patients. This was found to be linked with SLE disease activity in general and renal involvement in particular.

\section{References}

[1] Duarte C., Couto M., Ines L., Liang M. H. Epidemiology of systemic lupus erythematosus. In: Lahita R. G., Tsokos G., Buyon J., Koike T., editors. Systemic Lupus Erythematosus. 5th. London, UK: Elsevier; 2011. pp. 673-696.

[2] Kistler AD. Lupusnephritis. Ther Umsch. 2015; 72(3):171177.

[3] Muñoz LE, Lauber K, Schiller M, Manfredi AA, Herrmann M The role of defective clearance of apoptotic cells in systemic autoimmunity. Nat Rev Rheumatol. 2010; 15: 280-289. doi: 10.1038/nrrheum.2010.46.

[4] Shao WH, Cohen PL. Disturbances of apoptotic cell clearance in systemic lupus erythematosus. Arthritis Res Ther. 2011; 15: 202. doi: 10.1186/ar3535.

[5] Alberto de Zubiria Salgado, Catalina Herrera-Diaz. Lupus Nephritis: An Overview of Recent Findings. Autoimmune Dis. 2012; 2012: 849684. Published online 2012 March 22. doi: $10.1155 / 2012 / 849684$.

[6] Tackey E, Lipsky PE, Illei GG. Rationale for interleukin-6 blockade in systemic lupus erythematosus. Lupus.2004; 13 (5):339-343.

[7] Sanz AB, Sanchez-Nino MD, Ortiz A. TWEAK, a multifunctional cytokine in kidney injury. Kidney Int.2011; 80 (7):708-718.

[8] Kitani A, Hara M, Hirose T, et al. Heterogeneity of B cell responsiveness to interleukin 4, interleukin 6 and low molecular weight B cell growth factor in discrete stages of B cell activation in patients with systemic lupus erythematosus. Clin Exp Immunol. 1989; 77 (1):31-36.

[9] Kitani A, Hara M, Hirose T, et al. Autostimulatory effects of IL-6 on excessive B cell differentiation in patients with systemic lupus erythematosus: analysis of IL-6 production and IL-6R expression. Clin Exp Immunol.1992; 88 (1):75-83.

[10] Tilg H, Trehu E, Atkins MB, Dinarello CA, Mier JW. Interleukin-6 (IL-6) as an anti-inflammatory cytokine: induction of circulating IL-1 receptor antagonist and soluble tumor necrosis factor receptor p55. Blood.1994; 83 (1):113-118.

[11] Dai C, Wang H, Sung SS, Sharma R, Kannapell C, Han W, Wang Q, Davidson A, Gaskin F, Fu SM. Interferon alpha on NZM2328.Lc1R27: enhancing autoimmunity and immune complex-mediated glomerulonephritis without end stage renal failure. Clin Immunol. 2014 Sep; 154 (1):66-71. doi: 10.1016/j.clim.2014.06.008. Epub 2014 Jun 27.

[12] McCarthy EM, Smith S, Lee RZ, Cunnane G, Doran MF, Donnelly S, Howard D, O'Connell P, Kearns G, Ní Gabhann J, Jefferies CA. The association of cytokines with disease activity and damage scores in systemic lupus erythematosus patients. Rheumatology (Oxford). 2014 Sep; 53(9):1586-1594. doi: 10.1093/rheumatology/ket428. Epub 2014 Apr 4. 
[13] Yougbaré I, Keravis T, Abusnina A, Decossas M, Schall N, Muller S, Lugnier C. Cyclic GMP catabolism up-regulation in $\mathrm{MRL} / \mathrm{lpr}$ lupus-prone mice is associated with organ remodeling. Biochim Biophys Acta. 2014 Jul; 1842 (7):916926. doi: 10.1016/j.bbadis.2014.03.001. Epub 2014 Mar 12.

[14] Tan EM, Cohen AS, Fries JF, Masi AT, McShane DJ, Rothfield NF, et al. The 1982 revised criteria for the classification of systemic lupus erythematosus. Arthritis Rheum 1982; 25: 1271-1277.

[15] Hay EM, Bacon PA, Gordon C Isenberg DA, Maddison P, Snaith ML, et al. The BILAG index: a reliable and valid instrument for measuring clinical disease activity in systemic lupus erythematosus. QJ Med 1993; 86: 447-458.

[16] Schuett H, Luchtefeld M, Grothusen C, et al. How much is too much? Interleukin-6 and its signalling in atherosclerosis. Thromb Haemost 2009; 102: 215-222.

[17] Petri M, Kasitanon N, Lee SS, Link K, Magder L, Bae SC, et al. Systemic lupus international collaborating clinics renal activity/response exercise: Development of a renal activity score and renal response index. Arthritis \& Rheumatism 2008; 58 (6), 1784-1788

[18] Sabry A, Sheashaa H, El-Husseini A, Mahmoud K, Eldahshan KF, George SK, Abdel-Khalek E, El-Shafey EM, Abo-Zenah H. Cytokine. Proinflammatory cytokines (TNF-alpha and IL-6) in Egyptian patients with SLE: its correlation with disease activity. 2006 Aug; 35 (3-4):148-153. Epub 2006 Oct 5.

[19] Zhu LJ1, Yang X, Yu XQ. Anti-TNF-alpha therapies in systemic lupus erythematosus. J Biomed Biotechnol. 2010; 2010: 465898. doi: 10.1155/2010/465898.

[20] Kasitanon N, Magder LS, Petri M. Predictors of survival in systemic lupus erythematosus. Medicine (Baltimore). 2006; 85:147.

[21] Lazennec G, Richmond A: Chemokines and chemokine receptors: New insights into cancer-related inflammation. Trends Mol Med. 2010; 16: 133-144.

[22] Segerer S, Nelson PJ, Schlondorff D: Chemokines, chemokine receptors, and renal disease: From basic science to pathophysiologic and therapeutic studies. J Am Soc Nephrol. 2000; 11: 152-176.

[23] Dienz O, Rincon M. The effects of IL-6 on CD4 T cell responses. Clin Immunol. 2009; 130: 27-33.

[24] Li Y, Tucci M, Narain S, et al. Urinary biomarkers in lupus nephritis. Autoimmun Rev. 2006; 5: 383-388.

[25] Okada M, Kitahara M, Kishimoto S, Matsuda T, Hirano T, Kishimoto T. IL-6/BSF-2 functions as a killer helper factor in the in vitro induction of cytotoxic T cells. J Immunol. 1988; 141: $1543-1549$.

[26] Tanigawa T, Nicola N, McArthur GA, Strasser A, Begley CG. Differential regulation of macrophage differentiation in response to leukemia inhibitory factor/oncostatinM/interleukin-6: the effect of enforced expression of the SCL transcription factor. Blood. 1995; 85: 379-390.

[27] Richards HB, Satoh M, Shaw M, Libert C, Poli V, Reeves WH. Interleukin 6 dependence of anti-DNA antibody production: evidence for two pathways of autoantibody formation in pristane-induced lupus. J Exp Med.1998; 188: 985-990.
[28] Tackey E, Lipsky PE, Illei GG. Rationale for interleukin-6 blockade in systemic lupus erythematosus. Lupus.2004; 13 (5):339-343.

[29] Rovin BH1, Parikh SV2. Lupus nephritis: the evolving role of novel therapeutics. Am J Kidney Dis. 2014 Apr; 63 (4):677-90. doi: 10.1053/j.ajkd.2013.11.023. Epub 2014 Jan 7.

[30] Crow MK. Interferon pathway activation in systemic lupus erythematosus. Curr Rheumatol Rep. 2005; 7: 463-468.

[31] Wei ZF, Jiao XL, Wang T, Lu Q, Xia YF, Wang ZT, et al. Norisoboldine alleviates joint destruction in rats with adjuvant-induced arthritis by reducing RANKL, IL-6, PGE $_{2}$, and MMP-13 expression. Acta Pharmacol Sin. 2013; 34: 403413.

[32] Grondal G, Gunnarsson I, Ronnelid J, Rogberg S, Klareskog L, Lundberg I: Cytokine production, serum levels and disease activity in systemic lupus erythematosus. Clin Exp Rheumatol 2000, 18:565-570.

[33] Hagiwara E, Gourley MF, Lee S, Klinman DK: Disease severity in patients with systemic lupus erythematosus correlates with an increased ratio of interleukin-10: interferongamma-secreting cells in the peripheral blood. Arthritis Rheum 1996, 39:379-385.

[34] Esposito P, Balletta MM, Procino A, Postiglione L, Memoli B: Interleukin-6 release from peripheral mononuclear cells is associated to disease activity and treatment response in patients with lupus nephritis. Lupus 2009, 18: 1329-1330.

[35] Liang B, Gardner DB, Griswold DE, Bugelski PJ, Song XYR. Anti-interleukin-6 monoclonal antibody inhibits autoimmune responses in a murine model of systemic lupus erythematosus. Immunology.2006; 119: 296-305.

[36] Ball EM, Gibson DS, Bell AL, Rooney MR. Plasma IL-6 levels correlate with clinical and ultrasound measures of arthritis in patients with systemic lupus erythematosus. Lupus. 2014; 23(1):46-56.

[37] Iwano M, Dohi K, Hirata E, et al. Urinary levels of IL-6 in patients with active lupus nephritis. Clinical Nephrology. 1993; 40 (1):16-21.

[38] Herrera-Esparza R, Barbosa-Cisneros O, Villalobos-Hurtado R, Avalos-Díaz E. Renal expression of IL- 6 and TNF $\alpha$ genes in lupus nephritis. Lupus. 1998; 7 (3):154-158.

[39] Yung S1, Tsang RC, Sun Y, Leung JK, Chan TM. Effect of human anti-DNA antibodies on proximal renal tubular epithelial cell cytokine expression: implications on tubulointerstitial inflammation in lupus nephritis. Journal of the American Society of Nephrology. 2005; 16 (11):32813294.

[40] Peterson E, Robertson AD, Emlen W. Serum and urinary interleukin-6 in systemic lupus erythematosus.Lupus. 1996; 5 (6):571-575.

[41] Ryffel B, Car BD, Gunn H, Roman D, Hiestand P, Mihatsch MJ. Interleukin-6 exacerbates glomerulonephritis in (NZB $\times$ NZW) F1 mice. Am J Pathol. 1994; 144 (5):927-937.

[42] Yung S, Cheung KF, Zhang Q, Chan TM. Anti-dsDNA antibodies bind to mesangial annexin II in lupus nephritis. Journal of the American Society of Nephrology. 2010; 21(11):1912-1927. 